\title{
Educational Interventions in Emergency Contexts Editorial Prefece
}

\author{
Francesca Cuzzocrea $^{1, *}$, Antonella Nuzzaci \\ ${ }^{1}$ Department of Human and Social Sciences, University of Messina, Messina, Italy \\ ${ }^{2}$ Department of Human Sciences, University of L'Aquila, Aquila, Italy
}

\begin{abstract}
The aim of this special issue is to foster state-of-the-art research in this topic and to collect proposals for support for parents and teachers to people with difficulties that have been experimentally verified from first principles of research methodology and scientific basis of experimental pedagogy.
\end{abstract}

Keywords Education, Family, School, Emergency contexts, Territory, Italian Educational Research

\section{Introduction}

The main purpose of this special issue is to present some educational interventions aimed at families and the school contexts with a multidisciplinary view. Scientific research has clearly highlighted the role of the family and school in promoting the development of children. Adequate support the parents of a child with disabilities is one of the most important aspects to ensure the development of educational skills of the parents. Similarly, the scientific literature confirms the importance of properly train teachers of children with special needs, literacy difficulties and lower socio-economic status.

Provide families and school specific and effective training is certainly one of the main tasks of pedagogy, educational psychology and special education. These disciplines can help to better calibrate actions in all those emergency situations where it is necessary to act with educational interventions which, to be effective, must be guided by research.

It's also true that, in many cases, re-education-training proposals were not supported by adequate experimental data that verify the effectiveness of short, medium and long term.

The aim of this special issue is to foster state-of-the-art research in this topic and to collect proposals for support for parents and teachers to people with difficulties that have been experimentally verified from first principles of research methodology and scientific basis of experimental pedagogy.

The most critical, stressful and problematic period, for families of children with disabilities is what sees them engaged in the process of adaptation, the period, that is, where all their resources, such as personal or family history,

* Corresponding author:

fcuzzocrea@unime.it (Francesca Cuzzocrea)

Published online at http://journal.sapub.org/edu

Copyright (C) year Scientific \& Academic Publishing. All Rights Reserved are required; the period in which they need a great support.

For this reason, from many years, group of research belonging to University of Messina are working in order to planning interventions to accompany the couple during the transition to parenthood, especially in cases where it is considered least likely diagnosis of a disability. And, even in this case, a coordination of all the actors involved (both in health care, both in education and rehabilitation) is considered essential for the functionality of the intervention.

On the other hand, the researches of University of Aquila, from many years are working on specific Emergency Contexts underlining as catastrophes events require exceptional logistic, health, psychological, educational and social intervention. On the other hand, they proposed an educational cultural collaboration illustrating the advantages and the obstacles required.

\section{Special Issue Organization}

This Special Issue was organized in two different parts. More specifically the first three contributes were focused on families and specialist that have the responsibility on helping disabled children.

In the second part of this special Issue were taken in consideration others educational contexts. The need for psychological and educational support for students after a disaster is well documented in literature and this part is focusing on the role of the community following a disaster that has been shown to be important.

\subsection{First Contribute}

The aim of the paper presented by Cuzzocrea et al. is to focus on adaptation processes that families of disabled children must realize for a correct beginning of the entire system. In particular, difficulties that parents must face in order to manage maladaptive and non compliant behaviours 
were analysed. A Behavioural Parent Training was proposed to parents of non compliance children with intellectual disabilities and to parents of non compliance children but with a typical development.

The validity and effectiveness of these interventions of parent training proved for decades by those who work in this area, should make us reflect on whether to support systematic use of rehabilitative interventions on children, this type of training support for parents. However, furthers research should be take into account the conditions of applicability in different contexts and the variability of its related conditions who sees the families of children with disabilities involved in the adaptation process, in which the support it must be expressed in different forms. This dimension is essential for the functionality of the interventions.

\subsection{Second Contribute}

The aim the research presented by Oliva et al. was to explore parent-child interaction processes. In particularly, it was to verify the efficacy of behavioural parent training for parents with mentally retarded and non-compliant children. The programme proposed try to change parents' misguided beliefs about their children's non-compliant behaviours, improving the parenting style and encouraging more functional family interactions, in order to reduce parental stress and increase parenting skills.

Results indicate that child behavior, parent stress, and parental perceptions were more adaptive at post-training than before treatment and families in the treatment group showed an advantage over control families. This experimental research underlines the need to engage families with difficult children in continuing care interventions which may help parents better maintain their skills and adjust them in response to developmental change.

The parent training tested suggests several implications for future development of parent training and subsequent research. First, more emphasis should be placed on assessment, particularly when related to children with complex disabilities. Second, it would be interesting in future studies to assess the effect of this training on children's behavior in other contexts such as the school.

\subsection{Third Contribute}

This paper presented by Zampino et al. reported a research aimed at implementing a training program to promote compliance during the rehabilitation intervention in parents of children with intellectual disabilities. There were evaluating the anxiety-depressive states of the parents, and look for the variables that can affect the proper functioning of the family as an agent of change.

The training's goal was to implement a training program to promote compliance during the rehabilitation intervention in parents of children with intellectual disabilities. It is authors' opinion the importance to increase parental observational ability, teaching them how to discriminate between dysfunctional behaviors related to educational practices and those related to disabilities, but also to providing them with the basic knowledge concerning rehabilitation techniques used by professionals.

This study underlines the importance of carrying out of psycho educational support to families. Model of parenting skills, increased effective family interactions, increased parental compliance to the rehabilitation intervention, identifying the psychological resources and modulate the states of parental distress are the goals that a training program should work towards. However, it is necessary to encourage the rehabilitation team to work in collaboration with parents, children, teachers and other health professionals.

\subsection{Fourth Contribute}

The paper proposed by Isidori presents the results of a study carried out on University students in post-emergency conditions in the city of L'Aquila, two years after the devastating 2009 earthquake, in order to individuate the consequences that this catastrophic event had on student motivation and academic performance. This paper aims at outlining aspects of learning and studying in critical situations and defining a teaching intervention model to be adopted under such conditions. This research analysed the difference in academic performance between students who had experienced the earthquake and those who had only experienced the post- earthquake situation.

This study revealed that motivation towards learning, defined mainly as time and commitment dedicated to a task is greater in the group of students who experienced the 2009 earthquake.

The results underlined as educational interventus during emergency situations must focus on helping young people in overcoming learned helplessness, developing control and coping strategies and carrying out recognition of the event experienced focusing on emotional-cognitive dimensions.

With these aims, social networks provide people with positive experiences and stable roles, and, independently of individual stress, social support seems to directly influence mental health, reducing negative consequences of the stressful event.

\subsection{Fifth Contribute}

The objective of the research presented by Nuzzaci was to develop, test, evaluate and validate a model of guided tour with public school. The study covered the trial in a deeper and broader discourse on the relationship between school museum in order to study the phenomena related to the link between school and cultural institutions. More specifically, the relations established between teachers and operators, are characterized by a circumstantial and random collaboration, which cannot guarantee the efficiency of the structures. It's important to propose a social re-contextualization of teaching, in order to establish "a territory of education and teaching". The research reported, in a first stage, an exploratory study with the aim to understand the 
relationships between museum and education, between school and museum didactics. In a later stage, it is proposed an experimental study focus on learning and memory of the experience of the guided visit to a demo-ethno anthropological museum: the National Museum of Arts and Popular Traditions of Rome. The author proposed a wider research programme with the idea to evaluate a model of guided tour addressed to a school audience, which envisaged the experiment within a wider and more articulated debate on the collaborative practices in local partnership and with the aim to validate a didactical methodology to be used in archaeological museum contexts in order to improve the educational co-design forms and the collaboration actions between institutions. Everything with the purpose to promote joint actions between school-museum-local bodies aimed at enhancing the strengthening of children, teenagers and adults' alphabetical skills through the regular and aware fruition of cultural heritage. The heritage fruition here is intended as a cultural right allowing the access of individuals to symbolic systems brought by culture.

\section{Conclusions}

This special issue is concerned with the definition of "emergencies" in the field of educational research and shows some examples of how it is changing the role of the Research in Education in representing and constituting this field.

The "educational research" is a field of research and of policy and practice characterized to a methodological eclecticism which can be applied to the huge variety of procedures, theories, epistemologies and topics legitimately utilised and addressed within the field of educational. At the same time, the special issue argues the need to internationalize and globalise the educational research image. This call is located within a broader argument suggesting the need for a new educational imaginary to frame educational policy and practice and the contribution that educational theory and research might make to the society. In relation to this, the studies presented the difficulties that social representations of such a new imaginary might entail for educational research, given the variety of its themes and huge diversity of its interests, also in an interdisciplinary, multidisciplinary and trans-disciplinary.

\section{ACKNOWLEDGEMENTS}

These articles want to be an example of how the Italian educational research is trying to align itself with the best international research areas dealing with "emerging" and "emergencies".

The authors would like to thank the journal that hosted their contributions but also the Italian masters that through scientific societies are bringing all the educational research (historical, theoretical, empirical etc.) at the highest level.

Our special thanks goes to Prof. Luciano Galliani, President of Società Italiana di Ricerca Educativa (SIRD), Prof. Pier Cesare Rivoltella, President of Società Italiana di Ricerca sull'Educazione Mediale (SIREM), Prof. Umberto Margiotta, President of Società Italiana di Ricerca Educativa e Formativa (SIREF), Prof. Luigi D'Alonzo, President of Società Italiana di Pedagogia Speciale (SIPeS), Prof. Michele Corsi, President of Società Italiana di Pedagogia (SIPED), Prof. Giuseppe Trebisacce, President of the Centro Italiano per la Ricerca Storico-Educativa (CIRSE). 